\title{
Associação Entre Nível de Atividade Física, Indicadores de Saúde e Qualidade de Vida de Idosos da Universidade da Maturidade, Palmas-TO
}

\section{Association Between Physical Activity Level, Health Indicators and Quality of Life of the Elderly of the University of Maturity, Palmas-TO}

Aline Monique Galdiano Silva Baptista¹, Neilton Araújo de Oliveira², Luiz Sinésio Silva $\mathrm{Neto}^{3}$

\section{RESUMO}

Objetivo: verificar os níveis de atividade física, indicadores de saúde e qualidade de vida dos idosos da UMA/UFT, em Palmas-TO. Metodologia: participaram deste estudo 59 idosos, de ambos os sexos, participantes da Universidade da Maturidade; que se submeteram à avaliação do estado nutricional pela MAN; avaliação do nível de atividade física pelo IPAQ- versão curta; avaliação da qualidade de vida (QV) pelo SF36 e avaliação da força de preensão palmar (FPP) pelo dinamômetro Jamar. Os dados foram apresentados por meio da estatística descritiva e Coeficiente de Correlação de Pearson. O software SPSS, versão 19,0, foi utilizado para realização de todas as análises, adotando-se a significância de 95\%. Resultados: Dos 59 idosos, 14(23,72\%) homens e $45(76,28 \%)$ mulheres, média de idade de $67,77 \pm 7,19$ anos e $66,10 \%$ deles apresentaram nível insuficiente de atividade física (AF). Foi encontrada significância estatística entre o nível de AF e as variáveis $\operatorname{FPP}(p=0,019)$, sexo $(p=0,006)$ e três domínios da QV: CF $(0,048)$, Dor $(0,049)$ e VIT $(0,047)$. A variável FPP correlacionouse positiva e significativamente com os domínios CF $(p=0,011)$ e Dor $(p=0,03)$ do SF36. Conclusão: Os idosos apresentaram níveis satisfatórios de QV relacionada à saúde, apesar do baixo nível de atividade física, baixa condição socioeconômica e condições crônicas de saúde, demonstrando o caráter multidimensional da QV. Por outro lado, um estilo de vida mais ativo sugere uma melhor QV e melhor FPP, e a FPP, por sua vez, mostrou-se um importante fator nos determinantes da QV.

Palavras-chave: Envelhecimento. Nível de atividade física. Qualidade de vida.

\section{ABSTRACT}

Objective: To verify the association between physical activity level, health indicators and quality of life variables in the UMA / UFT elderly, in Palmas-TO. Methodology: 59 elderly people, of both sexes, participating in the University of Maturity participated in this study; who underwent nutritional status assessment by MAN; assessment of the level of physical activity by IPAQ - short version; evaluation of the quality of life (QoL) by the SF-36 and assessment of palmar grip strength (FPP) by the Jamar dynamometer. Data were presented through descriptive statistics and Pearson's Correlation Coefficient. The SPSS software, version 19.0, was used to perform all the analyzes, adopting the significance of 95\%. Results: Of the 59 elderly, 14 (23.72\%) men and 45 (76.28\%) women, mean age of $67.77 \pm 7.19$ years and $66.10 \%$ of them had insufficient level of physical activity. Statistical significance was found between the level of PA and the variables FPP $(p=0.019)$, sex $(p=0.006)$ and three domains of QoL: CF $(0.048)$, Pain (0.049) and VIT (0.047). The FPP variable correlated positively and significantly with the CF $(p=0.011)$ and Pain $(p=0.03)$ domains of SF-36. Conclusion: The elderly presented satisfactory levels of health-related QoL despite the low level of physical activity, low socioeconomic status and chronic health conditions, demonstrating the multidimensional nature of QoL. On the other hand, a more active lifestyle suggests a better QoL and better FPP, and FPP, in turn, has been an important factor in the determinants of QoL.

Keywords: Aging. Physical activity level. Quality of life.
${ }^{1}$ Mestrado em Ciências da Saúde da Universidade Federal do Tocantins.

E-mail:

alinegaldiano@yahoo.com.br

${ }^{2}$ Doutorado em Ensino de Biociências e Saúde pelo Instituto Oswaldo Cruz, Professor Adjunto da Universidade Federal do Tocantins.

${ }^{3}$ Doutorado em Ciência Tecnologias e Saúde pela Universidade de Brasilia, Brasil e Professor Adjunto da Universidade Federal do Tocantins. 


\section{INTRODUÇAOO}

Segundo declaração da Organização Mundial de Saúde, de 2014, nas próximas décadas a população mundial com mais de 60 anos vai chegar a 2 bilhões até 2050 . É possível observar um novo cenário de saúde se apresentando atualmente, resultado de transformações como o aumento do número de idosos e o desenvolvimento científico e tecnológico (VERAS e CALDAS, 2004).

Como os indivíduos estão vivendo mais, a grande questão desafiadora da contemporaneidade é incorporar mais saúde e maior qualidade de vida a essa longevidade (SILVA et al., 2012; ALMEIDA et al., 2014 e LIMA COSTA e VERAS, 2003; VERAS, 2009). Embora as pessoas estejam vivendo mais, não significa necessariamente que elas estejam mais saudáveis (OMS, 2014). Outra questão relevante é o fato de que à medida que aumenta a idade cronológica, as pessoas tornam-se menos ativas e sua independência funcional diminui (BRASIL, 2011), por outro lado, um estilo de vida ativo tem impacto positivo na longevidade, proporcionando ao idoso maior independência no dia a dia e maior qualidade de vida (MATSUDO, 2001).

A saúde da população idosa é multidimensional (LIMA-COSTA, 2011). Nesse sentido, se faz importante conhecer as condições de vida, de saúde, econômicas e sociais dos idosos para entender o fenômeno do envelhecimento e contribuir para a formulação de políticas públicas voltadas à saúde dessa população (MIRANDA, MENDES e SILVA, 2016; LEBRÃO et al., 2005).

Um importante fenótipo de qualidade de vida dos idosos é a força muscular. A força de preensão palmar (FPP) possui relevância clínica, de qualidade e função muscular. A baixa FPP é um importante preditor de limitada mobilidade, fraqueza muscular, mortalidade e qualidade de vida em idosos (SILVA NETO et al., 2012). Além disso, é um exame não invasivo, de baixo custo e fácil implementação na pratica clínica e saúde pública.

Nesse sentido, o projeto de extensão Universidade da Maturidade (UMA) da Universidade Federal do Tocantins (UFT), através de sua proposta pedagógica, voltada a atender às necessidades das pessoas acima de 45 anos (UMA, 2017), representa uma importante ferramenta de atenção à saúde do idoso. Este trabalho teve como objetivo verificar os níveis de atividade física, indicadores de saúde e qualidade de vida dos idosos da Universidade da Maturidade (UMA) da Universidade Federal do Tocantins (UFT). 


\section{MATERIAIS E METODOS}

Essa pesquisa é de natureza observacional, do tipo correlacional, transversal e com enfoque quantitativo, foi realizada com 59 idosos, de ambos os sexos, com idade média de $67,77 \pm 7,19$ (variando entre 60 e 86 anos), todos participantes do Projeto de Extensão Universidade da Maturidade (UMA) da Universidade Federal do Tocantins (UFT), Palmas - TO, Brasil. Os critérios de inclusão adotados foram indivíduos com faixa etária igual ou superior a 60 anos, de ambos os sexos, matriculados na UMA/UFT no ano de 2017 e escore maior que 17 no Mini-Exame do Estado Mental (MEEM). E os critérios de exclusão foram idosos com diagnóstico ortopédico e/ou neurológico que pudesse alterar a execução dos testes, como: fratura recente (menos de um ano) no membro superior, dificuldade de deambulação, Doença de Parkinson, déficit cognitivo, alteração de tônus e doença crônica não controlada. O presente estudo foi submetido e aprovado pelo Comitê de Ética em Pesquisa da UFT, parecer número 2.371.972, de acordo com a Resolução CNS 466/2012 e a coleta de dados realizada após a assinatura do termo de consentimento livre e esclarecido pelos participantes.

\section{Avaliação do Nível de Atividade Física}

$\mathrm{Na}$ caracterização do nível de atividade física $(\mathrm{AF})$ foi utilizado o Questionário Internacional de Atividades Físicas (IPAQ - International Physical Activity Questionnaire) versão curta, validado para a população idosa brasileira. A partir desse questionário foi possível estimar o tempo semanal gasto em atividades físicas de intensidade moderada e vigorosa, em diferentes contextos do cotidiano, como: trabalho, transporte, tarefas domésticas e lazer, e ainda o tempo despendido em atividades passivas, realizadas na posição sentada; e assim classificar o nível de atividade física em muito ativo, ativo, insuficientemente ativo e sedentário (BENEDETTI et al., 2007). Na sequência, e de acordo com essa classificação, os sujeitos foram relacionados em dois grupos: "Insuficientemente Ativos" (integrado pelos indivíduos sedentários e os insuficientemente ativos) e "Suficientemente Ativos" (integrado por indivíduos ativos e muito ativos), para fins de associação com as demais variáveis. 


\section{Avaliação dos indicadores de saúde - Variáveis cognitivas, socioeconômicas,} antropométricas, nutricionais e força muscular

O MEEM foi aplicado para a triagem cognitiva seguindo as recomendações de Brucki (2013) para seu uso no Brasil. Adicionalmente foi realizada a coleta de dados sociodemográficos; as patologias prévias foram obtidas por meio do autorrelato e as informações de uso de medicamentos a partir do inventário de medicamentos, seguindo o modelo utilizado na Avaliação Geriátrica Ampla (COSTA, 2005). A Mini Avaliação Nutricional (MAN) foi realizada para avaliar o estado nutricional (Duarte, 2007). Na avaliação antropométrica foram coletados: peso, estatura, circunferências da cintura (CC), do quadril (CQ) e da panturrilha (CP), seguindo as recomendações de Brasil (2011). Para a classificação do IMC (peso/estatura2), adotou-se a recomendação de Lipschitz, (LIPSCHITZ, 1994). A força de preensão manual foi aferida com o dinamômetro Jamar, como recomendado pela Sociedade Americana dos Terapeutas de Mão. Os voluntários foram posicionados sentados, cotovelo fletido a $90^{\circ}$ e antebraço em posição neutra; realizaram três repetições de cinco segundos de força máxima, alternadamente, com intervalo de um minuto e considerada a medida de maior valor. Os valores de normalidade adotados foram: $\geq 20 \mathrm{kgf}$ para as mulheres e $\geq 30 \mathrm{kgf}$ para os homens (CRUZ-JENTOFT et al., 2010).

\section{Avaliação da Qualidade de Vida}

A qualidade de vida relacionada à saúde (QVRS) foi medida utilizando-se o questionário SF-36; foram avaliadas questões a respeito da capacidade funcional, atividade física, dor, estado geral de saúde, vitalidade, aspectos sociais e emocionais e saúde mental. A pontuação para cada um dos 8 domínios varia de 0 (pior estado de saúde) a 100 (melhor estado de saúde). A classificação dos escores para cada domínio se deu após a ponderação inicial dos dados e posterior aplicação da fórmula para cálculo do raw scale; de acordo com o protocolo do questionário (CICONELLI, 1997).

\section{Análise dos dados}

Finalizada a coleta de todos os dados, os mesmos foram sistematizados em planilha do Excel e posteriormente armazenados num banco de dados do Statistical Package for Social Sciences (SPSS) para Windows, versão 19.0. As variáveis paramétricas e não paramétricas foram determinadas pelo teste de normalidade Shapiro-Wilk. Os dados foram 
apresentados por meio da estatística descritiva, utilizando-se os procedimentos de média e desvio-padrão. Para a associação das variáveis com o nível de atividade física, utilizouse o test t-Student e Qui-quadrado e a correlação de Pearson na associação de FPP com as variáveis da QV, adotando-se a significância de $95 \%$ :

\section{RESULTADOS}

Os resultados da caracterização da amostra foram apresentados de forma descritiva, considerando-se médias e desvios-padrão de todas as variáveis (tabela 1). Participaram deste estudo 59 idosos de ambos os sexos, com faixa etária compreendida entre 60 e 86 anos, média de $67,97 \pm 7,19$ anos de idade, sendo a grande maioria dos sujeitos do sexo feminino ( $n=45 ; 76,28 \%$ ), idade entre $60-69$ anos $(n=37 ; 62,72 \%)$, viúvos (as) ( $n=22$; $37,29 \%$ ), escolaridade nível fundamental ( $n=33 ; 55,93 \%$ ) e a renda familiar média de 2,32 $\pm 1,96$ salários mínimos. Os indivíduos insuficientemente ativos e sedentários representaram a maioria (66\%), em relação aos muito ativos e ativos (33,90\%).

A QVRS apresentou-se satisfatória em todos os domínios, cujas médias apresentadas foram superiores a 58 (tabela 2). Dentre eles destacam-se os aspectos sociais, com a maior média $(83,47 \pm 21,95)$ e a dor com a menor média $(58,65 \pm 25,44)$.

Dentre as patologias relatadas (figura 1) destacaram-se a hipertensão arterial $(n=$ $23 ; 38,98 \%)$ e artrose/artrite $(n=20 ; 33,89 \%)$.

O estado nutricional dos idosos avaliados, de forma geral mostrou-se adequado pela MAN e indicou sobrepeso pelo IMC (tabela 1). Porém, embora não tenha dado resultado estatístico, vale destacar que indivíduos com risco nutricional e sobrepeso foram observados somente entre os idosos insuficientemente ativos (tabela 3 ).

$\mathrm{Na}$ associação de variáveis com o nível de AF (tabela 4), foi possível observar diferença estatística significativa em relação ao sexo $(p=0,006)$; $\operatorname{FPP}(p=0,019)$ e domínios da capacidade funcional (CF) ( $p=0,048)$, Dor $(0,049)$ e vitalidade (VIT) $(p=0,047)$ da QVRS. Entre os idosos insuficientemente ativos e sedentários, uma maior prevalência de mulheres $(87,2 \%)$ em relação aos homens $(12,8 \%)$ foi observada. Os indivíduos suficientemente ativos (muito ativos+ativos) apresentaram maior média de $\operatorname{FPP}(31,83 \pm 7,7 \mathrm{kgf})$ em relação aos idosos com nível insuficiente de AF (insuficientemente ativos+sedentários) $(27,76 \pm$ 4,86kgf) bem como melhores médias na CF, Dor e VIT.

Os resultados também sugerem uma associação entre a FPP e QVRS na população estudada (tabela 5), uma vez que apresentou correlação significativa com duas dimensões, CF $(p=0,011)$ e dor $(p=0,03)$. 
Tabela 1. Estatística descritiva (média \pm desvio-padrão) das variáveis analisadas dos idosos da Universidade da Maturidade/Universidade Federal do Tocantins, Palmas -TO, 2017.

\begin{tabular}{|c|c|}
\hline Variáveis & \\
\hline$N$ & 59 \\
\hline Idade (anos) & $67,77 \pm 7,19$ \\
\hline $60-69$ anos & $37(62,72 \%)$ \\
\hline $70-79$ anos & $16(27,11 \%)$ \\
\hline$\geq 80$ anos & $6(10,17 \%)$ \\
\hline Sexo & - \\
\hline Masculino & $14(23,72 \%)$ \\
\hline Feminino & $45(76,28 \%)$ \\
\hline Escolaridade & - \\
\hline Fundamental & $33(55,93 \%)$ \\
\hline Médio & $18(30,51 \%)$ \\
\hline Superior & $8(13,56 \%)$ \\
\hline Renda & $2,32 \pm 1,96$ \\
\hline Estado Civil & - \\
\hline Solteiro(a) & $9(15,25 \%)$ \\
\hline Casado (a) & $20(33,90 \%)$ \\
\hline Separado(a) & $8(13,56 \%)$ \\
\hline Viúvo(a) & $22(37,29 \%)$ \\
\hline MEEM & $24,32 \pm 2,54$ \\
\hline Patologias & $2,75 \pm 0,78$ \\
\hline Medicamentos & $2,56 \pm 2,02$ \\
\hline$\geq 5$ & $9(15,25 \%)$ \\
\hline MAN & $26,04 \pm 1,63$ \\
\hline Peso (kg) & $67,21 \pm 10,95$ \\
\hline Estatura(m) & $1,57 \pm 0,09$ \\
\hline IMC $\left(\mathrm{Kg} / \mathrm{m}^{2}\right)$ & $27,38 \pm 4,56$ \\
\hline $\mathrm{CC}(\mathrm{cm})$ & $91,32 \pm 10,30$ \\
\hline $\mathrm{CQ}(\mathrm{cm})$ & $103,22 \pm 8,59$ \\
\hline RCQ & $0,88 \pm 0,07$ \\
\hline CB (cm) & $31 \pm 3,30$ \\
\hline $\mathrm{CP}(\mathrm{cm})$ & $36,20 \pm 3,29$ \\
\hline FPP & $28,83 \pm 5,34$ \\
\hline IPAQ & - \\
\hline $\begin{array}{l}\text { Insuficientemente Ativo + } \\
\text { Sedentário }\end{array}$ & $39(66,10 \%)$ \\
\hline Muito Ativo + Ativo & $20(33,90 \%)$ \\
\hline
\end{tabular}

MEEM=MiniExame do Estado Mental;MAN=MiniAvaliação Nutricional;CC=circunferência cintura; $\mathrm{CQ}=$ cincurferência quadril; $\mathrm{RCQ}=$ Relação cintura quadril; $\mathrm{CB}=$ circunferência do braço; $\mathrm{CP}=$ circunferência panturrilha; $F P P=$ força preensão palmar; $I P A Q=$ Questionário Internacional de Avaliação Física 
Tabela 2. Estatística descritiva (média \pm Desvio padrão) dos domínios de Qualidade de Vida dos idosos da Universidade da Maturidade, Palmas-TO, 2017.

\begin{tabular}{cc}
\hline Variáveis & \\
\hline$N$ & 59 \\
CF & $75 \pm 21,11$ \\
AF & $78,81 \pm 31,76$ \\
Dor & $58,65 \pm 25,44$ \\
EGS & $62,44 \pm 15,95$ \\
VIT & $70,04 \pm 21,02$ \\
AS & $83,47 \pm 21,95$ \\
AE & $74,56 \pm 39,80$ \\
SM & $72,47 \pm 21,60$ \\
\hline
\end{tabular}

$\mathrm{CF}=$ Capacidade Funcional; $\mathrm{AF}=$ Aspectos Físicos; Dor=Dor; EGS= Estado Geral de Saúde; VIT= Vitalidade; $\mathrm{AS}=$ Aspectos Sociais; $\mathrm{AE}=$ Aspectos Emocionais; $\mathrm{SM}=$ Saúde Mental.

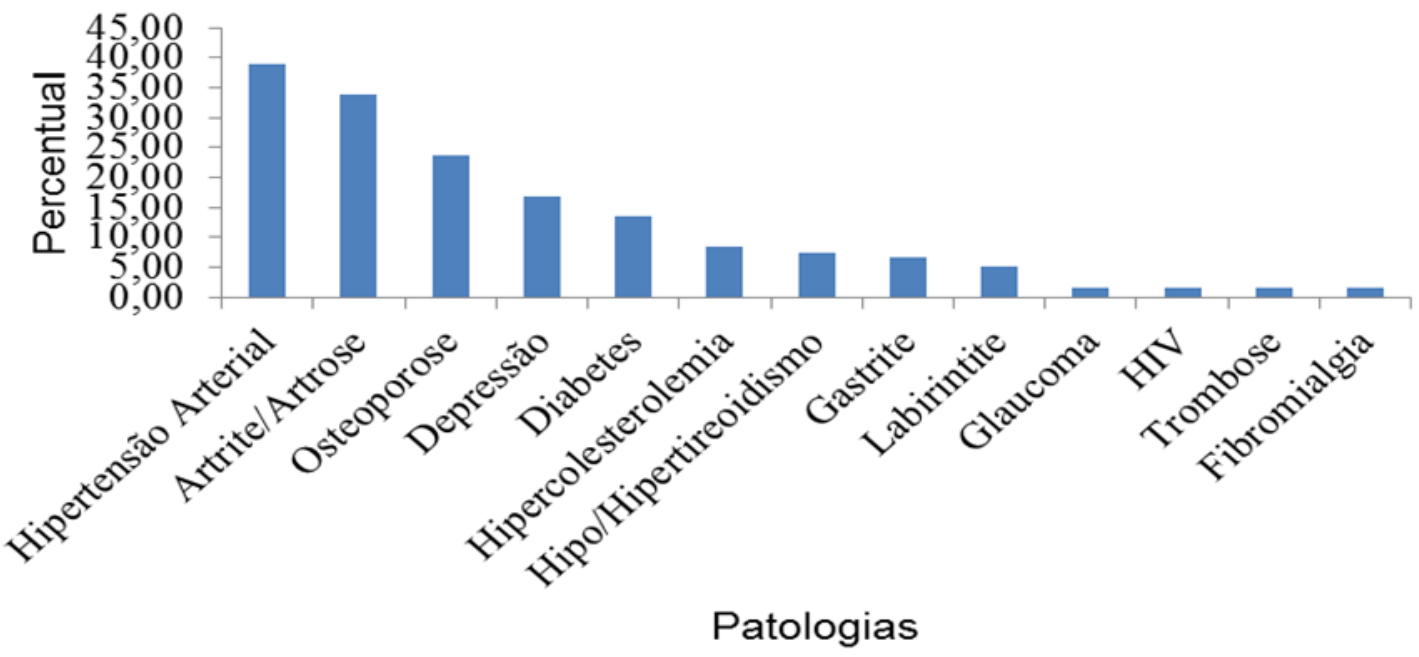

Figura 1. Percentual de patologias autorrelatadas pelos idosos da Universidade da Maturidade/Universidade Federal Tocantins, Palmas - TO, 2017. 
Tabela 3. Associação de variáveis com a classificação do nível de atividade física dos idosos da Universidade da Maturidade/Universidade Federal do Tocantins, Palmas-TO, 2017.

\begin{tabular}{|c|c|c|c|}
\hline Variáveis & $\begin{array}{l}\text { Insuficientemente } \\
\text { Ativo + Sedentário }\end{array}$ & $\begin{array}{l}\text { Muito Ativo } \\
\text { + Ativo }\end{array}$ & $\begin{array}{l}\text { Valor } \\
\text { de p }\end{array}$ \\
\hline $\mathbf{N}(\%)$ & $39(66,10 \%)$ & 20 (33,9\%) & - \\
\hline Idade (anos) & $67,08 \pm 7,21$ & $69,70 \pm 7,00$ & 0,126 \\
\hline $\begin{array}{c}\text { Sexo } \\
\text { Masculino (\%) } \\
\text { Feminino (\%) }\end{array}$ & $\begin{array}{r}- \\
12,8 \\
87,2\end{array}$ & $\begin{array}{r}- \\
45,0 \\
55,0\end{array}$ & $0,006^{\star}$ \\
\hline $\begin{array}{c}\text { Escolaridade } \\
\text { Ensino Fundamental } \\
\text { Ensino Médio } \\
\text { Ensino Superior }\end{array}$ & $\begin{array}{c}- \\
53,8 \\
28,2 \\
17,9\end{array}$ & $\begin{array}{c}- \\
60,0 \\
35,0 \\
5,0\end{array}$ & 0,353 \\
\hline Renda $^{1}$ & $\begin{array}{c}2,00 \text { IC } 95 \%: \\
1,76-2,65\end{array}$ & $\begin{array}{c}1,00 \text { IC95\%: } \\
1,23-3,87\end{array}$ & 0,301 \\
\hline $\begin{array}{c}\text { Estado Civil } \\
\text { Solteiro } \\
\text { Casado } \\
\text { Separado } \\
\text { Viúvo } \\
\end{array}$ & $\begin{array}{c}- \\
15,4 \\
33,3 \\
15,4 \\
35,9\end{array}$ & $\begin{array}{c}- \\
15,0 \\
35,0 \\
10,0 \\
40,0 \\
\end{array}$ & 1,000 \\
\hline MEEM & $24,38 \pm 2,43$ & $24,20 \pm 2,80$ & 0,794 \\
\hline $\begin{array}{c}\text { Patologias } \\
\text { Classificação Patologias } \\
<3 \\
\geq 3\end{array}$ & $\begin{array}{c}2,1 \pm 1,68 \\
- \\
61,5 \\
38,5\end{array}$ & $\begin{array}{c}2,4 \pm 1,98 \\
- \\
50,0 \\
50,0\end{array}$ & 0,396 \\
\hline $\begin{array}{c}\text { Medicamentos } \\
\text { Classificação de Medicamentos } \\
<5 \\
\geq 5\end{array}$ & $\begin{array}{c}2,66 \pm 1,96 \\
- \\
84,6 \\
15,4\end{array}$ & $\begin{array}{c}2,30 \pm 2,17 \\
- \\
85,0 \\
15,0\end{array}$ & $\begin{array}{l}0,354 \\
1,000\end{array}$ \\
\hline $\begin{array}{c}\text { MAN } \\
\text { Classificação MAN } \\
\text { Com Risco Nutricional } \\
\text { Sem Risco Nutricional }\end{array}$ & $\begin{array}{c}26,04 \pm 1,58 \\
- \\
10,3 \\
89,7\end{array}$ & $\begin{array}{c}26,05 \pm 1,77 \\
- \\
0,0 \\
100,0\end{array}$ & $\begin{array}{l}0,979 \\
0,289\end{array}$ \\
\hline
\end{tabular}


Tabela 4. Associação de variáveis com a classificação do nível de atividade física dos idosos da Universidade da Maturidade/Universidade Federal do Tocantins, Palmas-TO, 2017.

\begin{tabular}{|c|c|c|c|}
\hline Variáveis & $\begin{array}{l}\text { Insuficientemente } \\
\text { Ativo + Sedentário }\end{array}$ & $\begin{array}{l}\text { Muito Ativo } \\
+ \text { Ativo } \\
\end{array}$ & $\begin{array}{l}\text { Valor } \\
\text { de } p\end{array}$ \\
\hline Peso (kg) & $66,42 \pm 11,28$ & $\begin{array}{c}68,74 \\
\pm 10,38\end{array}$ & 0,446 \\
\hline Estatura (m) & $1,55 \pm 0,08$ & $1,59 \pm 0,09$ & 0,051 \\
\hline IMC $\left(\mathrm{kg} / \mathrm{m}^{2}\right)$ & $27,63 \pm 4,86$ & $26,91 \pm 3,97$ & 0,653 \\
\hline CC & $90,90 \pm 10,80$ & $92,15 \pm 9,45$ & 0,348 \\
\hline $\mathbf{C Q}$ & $103,5 \pm 9,33$ & $102,8 \pm 7,12$ & 0,766 \\
\hline $\mathbf{R C Q}$ & $0,86 \pm 0,07$ & $0,89 \pm 0,07$ & 0,276 \\
\hline CB & $30,82 \pm 3,27$ & $31,35 \pm 3,39$ & 0,563 \\
\hline $\mathrm{CP}$ & $36,00 \pm 3,23$ & $36,6 \pm 3,45$ & 0,628 \\
\hline FPP (Kg/f) & $27,76 \pm 4,86$ & $31,83 \pm 7,7$ & $0,019^{\star}$ \\
\hline CF & $70,92 \pm 22,17$ & $\begin{array}{c}84,21 \\
\pm 12,39\end{array}$ & $0,048^{*}$ \\
\hline AF & $80,13 \pm 30,45$ & $\begin{array}{c}80,26 \pm \\
30,71\end{array}$ & 0,875 \\
\hline Dor & $54,66 \pm 22,14$ & $69,88 \pm 22,19$ & $0,049^{\star}$ \\
\hline EGS & $61,31 \pm 16,01$ & $\begin{array}{c}64,65 \pm \\
16,02\end{array}$ & 0,349 \\
\hline VIT & $65,33 \pm 22,66$ & $\begin{array}{r}78,68 \\
\pm 13,42\end{array}$ & $0,047^{\star}$ \\
\hline AS & $82,69 \pm 21,58$ & $85 \pm 23,16$ & 0,605 \\
\hline AE & $75,20 \pm 38,79$ & $73,33 \pm 42,72$ & 0,961 \\
\hline SM & $72,04 \pm 21,72$ & $\begin{array}{c}76,70 \\
\pm 15,48\end{array}$ & 0,792 \\
\hline
\end{tabular}

IMC = Índice de Massa Corporal; FPP = Força de preensão palmar; $C F=$ Capacidade Funcional; $\mathrm{AF}=$ Aspectos Físicos; Dor=Dor; EGS= Estado Geral de Saúde; VIT= Vitalidade; $A S=A s p e c t o s$ Sociais; $\mathrm{AE}=$ Aspectos Emocionais; $\mathrm{SM}=$ Saúde Mental; * denota associação significativa $(p<0,005) ;{ }^{1}$ mediana com intervalo de confiança de $95 \%$. 
Tabela 5. Correlação entre níveis de atividade física e Força com variáveis de qualidade de vida dos idosos da Universidade da Maturidade/Universidade Federal do Tocantins, Palmas-TO, 2017.

\begin{tabular}{|c|c|c|c|c|c|c|c|c|c|c|c|}
\hline Variáveis & $\begin{array}{c}\text { Insuficientemente } \\
\text { Ativo + } \\
\text { Sedentário }\end{array}$ & $\begin{array}{l}\text { Muito } \\
\text { Ativo + } \\
\text { Ativo }\end{array}$ & FPP & CF & AF & Dor & EGS & VIT & AS & $\mathrm{AE}$ & SM \\
\hline $\begin{array}{c}\text { Insuficientemente } \\
\text { Ativo + } \\
\text { Sedentário }\end{array}$ & - & 0,180 & 0,849 & 0,789 & 0,094 & 0,373 & 0,619 & 0,313 & 0,968 & 0,929 & 0,819 \\
\hline $\begin{array}{l}\text { Muito Ativo + } \\
\text { Ativo }\end{array}$ & 0,180 & - & 0,352 & 0,166 & 0,533 & 0,553 & 0,096 & 0,230 & 0,490 & 0,441 & 0,554 \\
\hline FPP & 0,243 & 0,352 & - & $0,011^{*}$ & 0,299 & $0,030^{*}$ & 0,787 & 0,248 & 0,659 & 0,310 & 0,071 \\
\hline
\end{tabular}

$\mathrm{FPP}=$ força preensão palmar; $\mathrm{CF}=$ Capacidade Funcional; $\mathrm{AF}=$ Aspectos Físicos; Dor=Dor; EGS= Estado

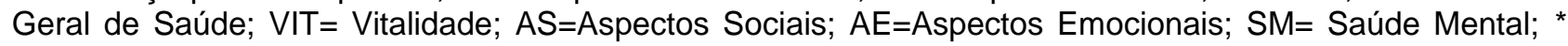
denota que a correlação foi significativa $(P<0.05)$

\section{DISCUSSÃO}

Dentre os principais achados desse estudo a FPP demonstrou ser um importante fenótipo de QVRS em idosos de acordo com o nível AF. A FPP está relacionada com a capacidade funcional e realização das atividades da vida diária (CRUZ-JENTOFT et al., 2010) e em idosos, o declínio de força muscular pode estar relacionado com maior incapacidade funcional e nível de dependência (SILVA et al., 2013). Homens e mulheres idosos com menor atividade física têm também menor massa muscular e maior prevalência de incapacidade física, diminuindo a aptidão e desempenho físico, o que os torna ainda mais inativos; podendo influenciar na autonomia, no bem-estar e na sua qualidade de vida (PICOLI et al., 2011; MOURA, 2008). Logo, o teste de FPP é utilizado como parâmetro para força muscular global e funcionalidade para idosos (MARTIN, NEBULONI e NAJAS; 2012).

De forma interessante, a variável FPP foi positiva e significativamente correlacionada com as dimensões CF e Dor da QVRS. Esses resultados confirmam as consequências positivas de melhores níveis de força com menor comprometimento da capacidade funcional bem como menor referência à dor pelos idosos; e corroboram com Silva Neto et al. (2012) que encontraram correlação positiva entre FPP e várias dimensões da QVRS, incluindo CF e Dor, em idosas da UMA/UFT.

A QVRS dos idosos mostrou-se satisfatória. Os domínios CF, Dor e VIT demonstraram associação positiva com maior nível de atividade física. A CF refere-se à eficiência do idoso em corresponder às demandas físicas do cotidiano na realização das atividades de vida diárias, desde atividades básicas até as ações mais complexas; e níveis mais baixos de CF podem interferir na eficiência das atividades cotidianas realizadas (CAMARA et al., 2008). Já a presença de dor constitui um fator negativo na QVRS nos idosos, uma vez que ela é 
um fator limitante nas atividades diárias e na funcionalidade (TOZIM et al., 2014). Estudos sugerem uma maior associação da dor crônica com o sexo feminino, a idade avançada e o baixo nível socioeconômico (SANTOS et al., 2015).

O presente estudo corrobora com a literatura, autores vêm destacando que a prática de exercícios físicos regulares durante a senescência tem sido amplamente descrita como um importante fator analgésico, capaz de impactar positivamente a qualidade de vida (TOZIM et al., 2014) preservando a saúde em geral, a vitalidade e função social (CAMPOS et al., 2014). Silva et al. (2012), colocam que a prática de qualquer atividade física é importante para a saúde dos idosos, contudo, quando esta atividade é praticada regularmente como exercício físico, melhores benefícios para a capacidade física são alcançados. Mas cabe ressaltar que a QVRS é multidimensional; vai além dos aspectos físicos e/ou funcionais, também tem relação direta com a autonomia e independência dos indivíduos.

O perfil sociodemográfico encontrado nesta pesquisa corrobora com Wichmann et al. (2013) que buscaram conhecer a representação da população idosa em grupos de convivência, e identificaram também um perfil dos participantes em sua maioria composto de idosos jovens, viúvos e predomínio de mulheres. O grupo de convivência da UMA/UFT, composto majoritariamente por mulheres e idosos de baixa escolaridade e renda, reflete a forte tendência da feminização da velhice (ZIMERMAN, 2000; VERAS, 2009) e sabendo que a baixa escolaridade compromete o acesso à educação em saúde (ANDRADE et al.,2014) e o nível socioeconômico exerce forte influência na saúde e qualidade de vida (LIMA-COSTA et al, 2003), a UMA/UFT parece constituir um ambiente positivo com oportunidade de potencializar os desenvolvimento pessoal e social e, assim contribuir para uma longevidade mais saudável e ativa desses idosos.

Entre os idosos insuficientemente ativos, foi possível observar uma maior prevalência de mulheres em relação aos homens, sugerindo a associação do menor nível de AF com o sexo feminino $(p=0,006)$. Essa diferença estatística pode ser resultante da diferente distribuição na proporção de mulheres e homens no grupo total de idosos pesquisados, o que poderia ter influenciado essa associação. Por outro lado, esse resultado difere do estudo de BENEDETTI, MAZO e BORGES et al. (2012) que avaliaram o nível de atividade física de idosos participantes de grupos de convivência e verificaram as mulheres idosas como mais ativas do que os homens idosos.

Estudos visando compreender os fatores do nível insuficiente de AF dos idosos da UMA/UFT devem ser incentivados. Nos idosos, em especial, o comportamento sedentário 
está associado negativamente à saúde e quando comparados com outras faixas etárias, são considerados os que mais despendem tempo em comportamento sedentário, o que aumenta o risco de mortalidade (REZENDE et al., 2014; MATTHEWS et al., 2008). Estar ativo nesse período da vida está associado positivamente a uma melhor qualidade de vida e independência na realização das atividades da vida diária (VAGETTI et al., 2017).

\section{CONSIDERAÇÓES FINAIS}

Os níveis satisfatórios de QVRS apresentados pelos idosos, apesar do baixo nível de atividade física, baixa condição socioeconômica e das condições crônicas de saúde, demonstraram o caráter multidimensional da QVRS. Por outro lado, sugere-se que um estilo de vida mais ativo seja capaz de impactar positivamente na QVRS, principalmente no que diz respeito a uma maior eficiência na realização das atividades de vida diárias e maior satisfação com a vida; e ainda garantir maior autonomia e independência com o incremento da FPP. Por sua vez, a FPP parece se apresentar como um importante preditor da QVRS. Deste modo, a prática de atividade física deve ser incentivada nos idosos; visando tanto à manutenção quanto a melhora da QVRS. E a partir dos resultados aqui apresentados é possível inferir ainda que a UMA/UFT representa uma importante ferramenta na promoção do envelhecimento com melhor qualidade de vida.

\section{REFERÉNCIAS}

ALMEIDA et al. Promoção da saúde, qualidade de vida e envelhecimento - A experiência do projeto "Em Comum-Idade: uma proposta de ações integradas para a promoção da saúde de idosos das comunidades de Viçosa-MG". Revista ELO - Diálogos em Extensão, v.3, n.2. 2014.

ANDRADE et al. Influência de fatores socioeconômicos na qualidade de vida de idosos hipertensos. Ciência \& Saúde Coletiva, v.19, n.8, 2014, p. 3497-3504.

BENEDETTI et al. Reprodutibilidade e validade do Questionário Internacional de Atividade Física (IPAQ) em homens idosos. Revista Brasileira Medicina Esporte, v. 13, n.1. 2007.

BENEDETTI, T.R.B.; MAZO, G.; BORGES, L.J. Condições de saúde e nível de atividade física em idosos participantes e não participantes de grupos de convivência de Florianópolis. Ciência \& Saúde Coletiva, v.17, n. 8, 2012, p. 2087-2093.

BRASIL. Coordenação-Geral de Alimentação e Nutrição, Departamento de Atenção Básica, Secretaria de Atenção à Saúde, Ministério da Saúde. Orientações para a coleta e análise de dados antropométricos em serviços de saúde: norma técnica do sistema de vigilância alimentar e nutricional - SISVAN. Brasília: Ministério da Saúde, 2011. (Série G. Estatística e Informação em Saúde). 
BRUCKI, et al. Sugestões para o uso do mini-exame do estado mental no Brasil. Arquivo de Neuropsiquiatria, v.61, n. 3-b, 2013, p. 777-781.

CAMARA et al. Capacidade funcional do idoso: formas de avaliação e tendências. Acta Fisiatrica, v. 15, n. 4, 2008, p. $249-256$.

CAMPOS, ACV et al. Aging, Gender and Quality of Life (AGEQOL) study: factors associated with good quality of life in older Brazilian community-dwelling adults. Health and quality of life outcomes, v.12, n. 1, 2014, p.1.

CICONELLI RM. Tradução para o português e validação do questionário genérico de avaliação de qualidade de vida medical outcomes study 36-item short-form health survey (SF-36) [Dissertação]. Säo Paulo: Universidade Federal de Säo Paulo; 1997.

COSTA, E.F.A. Avaliação Geriátrica Ampla (AGA). In: Liberman A, Freitas EV, Savioli Neto F, Taddei CFG. Diagnóstico e Tratamento em Cardiologia Geriátrica. São Paulo: Manole, 2005.

CRUZ-JENTOFT A et al. Sarcopenia: European consensus on definition and diagnosis. Age and Agein , v.39, 2010, p. 412-423.

DUARTE, A.C.G. Avaliação Nutricional- aspectos clínicos e laboratoriais. Capítulo $15-$ Avaliação Nutricional em Geriatria, p. 155. São Paulo: Editora Atheneu, 2007.

LEBRÃO, M.L. et al. Saúde, bem-estar e envelhecimento: o estudo SABE no Município de São Paulo. Revista Brasileira de Epidemiologia, v.8, n.2, 2005, p.127-41.

LIMA-COSTA, M.F.; BARRETO, S.; GIATTI, L., UCHOA. Desigualdade social e saúde entre idosos brasileiros: um estudo baseado na Pesquisa Nacional por Amostra de Domicílios. Caderno Saúde Pública, v.19, n.3, 2003, p.745-757.

LIMA-COSTA, M.F. et al. Tendências em dez anos das condições de saúde de idosos brasileiros: evidências da Pesquisa Nacional por Amostra de Domicílios (1998, 2003, 2008). Ciência \& Saúde Coletiva, v.16, n. 9. 2011.

LIMA COSTA, M.F e VERAS, R. Saúde pública e envelhecimento. Caderno Saúde Pública, v.19, n.3, 2003, p.700-701.

LIPSCHITZ, DA. Screening for nutritional status in the elderly. Primary Care, v.21, 1994, p.55-67.

MARTIN, F.G.; NEBULONI, C.C.; NAJAS, M.S. Correlação entre estado nutricional e força de preensão palmar em idosos. Revista Brasileira Geriatria Gerontologia, v.15, n.3, 2012, p.493-504.

MATTHEWS C.E. et al. Amount of time spent in sedentary behaviors in the United States, 2003-2004. American Journal Epidemiology, v.167, n.7, 2008, p.875-81.

MIRANDA, G.M.D.; MENDES, A.C.; SILVA, A.L. O envelhecimento populacional brasileiro: desafios e consequências sociais atuais e futuras. Revista Brasileira Geriatria Gerontologia, v.19, n.3, 2016, p.507-519. 
MOURA, P.M.L. Estudo da força de preensão palmar em diferentes faixas etárias do desenvolvimento humano. Brasília. Tese [Mestrado em Ciências da Saúde] - Universidade de Brasília; 2008.

ORGANIZAÇÃO MUNDIAL DA SAÚDE- OMS. Estatísticas globais de saúde. 2014. Disponível em < https://nacoesunidas.org > Acesso em 15/08/17.

PICOLI T.S.; FIGUEIREDO L.L.; PATRIZZI L.J. Sarcopenia e envelhecimento. Fisioterapia e Movimento, v. 24, n. 3, 2011, p. 455-462.

REZENDE L.F.M. et al. Sedentary behavior and health outcomes among older adults: a systematic review. BMC Public Health, v.14, n.333. 2014.

SANTOS F.A.S. et al. Prevalência de dor crônica e sua associação com a situação sociodemográfica e atividade física no lazer em idosos de Florianópolis, Santa Catarina: estudo de base populacional. Revista Brasileira de Epidemiologia, v.18, n.1, 2015, p. 23447.

SILVA NETO, L.S. et al. Associação entre sarcopenia, obesidade sarcopênica e força muscular com variáveis relacionadas de qualidade de vida em idosas. Revista Brasileira de Fisioterapia. 2012.

SILVA, M.F. et al. Relação entre os níveis de atividade física e qualidade de vida de idosos sedentários e fisicamente ativos. Revista Brasileira Geriatria e Gerontologia, v.15, n.4, 2012, p.635-642.

SILVA, N.A. et al. Força de preensão manual e flexibilidade e suas relações com variáveis antropométricas em idosos. Revista da Associação Médica Brasileira, v. 59, n.2, 2013, p. 128-135.

TOZIM, B.M. et al. Efeito do método Pilates na flexibilidade, qualidade de vida e nível de dor em idosos. ConScientia e Saúde, v.13, n.4, 2014, p.563-570.

UMA - UFT. Universidade da Maturidade/Universidade Federal do Tocantins. Disponível em< www.uft.deu.br/uma $>$ acesso em: 28/10/2017.

VAGETTI, G.C. et al. Associação do índice de massa corporal com a aptidão funcional de idosas participantes de um programa de atividade física. Revista de Geriatria e Gerontologia, v.20, n.2, 2017, p.216-227.

VERAS, R. Envelhecimento populacional contemporâneo: demandas, desafios e inovações. Revista Saúde Pública, v.43, n.3, 2009, p.548-54.

VERAS, R.P.; CALDAS, C.P. Promovendo a saúde e a cidadania do idoso: o movimento das universidades da terceira idade. Ciência \& Saúde Coletiva, v.9, n.2, 2004, p. 423-432.

WICHMANN et al. Grupos de convivência como suporte ao idoso na melhoria da saúde. Revista Brasileira Geriatria Gerontologia, v.16, n.4, 2013, p.821-832.

ZIMERMAN, G. L. Velhice - aspectos biopsicossociais. Porto Alegre: Artemed, 2000. 